\title{
Two and three coupled spins in magnetic field
}

\section{M.C. Baldiotti}

Universidade Estadual de Londrina

Departamento de Física, Caixa Postal 6001, CEP 86057-970, Londrina, P.R., Brazil

E-mail: baldiotti@uel.br

\section{D.M. Gitman*}

Universidade de São Paulo

Instituto de Física, Caixa Postal 66318, CEP 05508-090, São Paulo, S.P., Brazil

E-mail: gitmanedfn.if.usp.br

\section{M.M. Santos}

Universidade de São Paulo

Instituto de Física, Caixa Postal 66318, CEP 05508-090, São Paulo, S.P., Brazil

E-mail: marcelo.meireles.santoseusp.br

\begin{abstract}
We consider three spin systems in the parallel magnetic fields and present some of possible exact solutions. In addition, we discuss an analogue of the Rabi problem for three-spin system. In order to do this, we reduce the problem of three coupled spins, an 8-level system, to a problem of a 3-level system in an effective field. We explicitly construct the evolution operator of the system in two special cases: when the fields and interaction are the same in all the spins but with a arbitrary time-dependence of the fields, and when the spins are subjected to a circular magnetic field (the Rabi problem). Some applications of these results are discussed.
\end{abstract}

$3 d$ International Satellite Conference on Mathematical Methods in Physics - ICMP 2013

21 - 26 October, 2013

Londrina - PR (Brazil)

\footnotetext{
*Speaker.
} 


\section{Introduction}

Lattice models of interacting spins have applications in many physical problems, e.g., in condensed matter and quantum computation and information. Models, in which the interaction is restricted to the neighbors (e.g., the Ising model), attract a lot of attention due to their relative simplicity and, at the same time, due to their capability to describe a lot of important physical phenomena. For example, the two-dimensional square-lattice Ising model is used to describe phase transitions and so on. The model of a one-dimensional chain of spins, with finite elements, and each spin interacting only with its nearest neighbors, can be used to describe a system of coupled quantum dots. Recently this problem has attracted attention due to the possibility of such a system to be used in the implementation of quantum computations [1]. The system of two coupled quantum dots (two-qubit system) can be used to implement a universal quantum gate [2], i.e., any quantum algorithm can be performed using this system. The problem of three coupled spins has a direct application in the implementation of quantum error correction process [3]. Therefore, the study of three coupled quantum dots, in different configurations (linear and circular), besides of its theoretical interest, has a lot of practical application. It turns out that placing such system in an external electromagnetic field, one can control both interaction functions and transitions between possible quantum states of the system. The simplest case when magnetic fields applied to each spin of the system are parallel is already enough to implement any quantum gate [河]. The obtaining of a group of universal quantum gates demands the implementation of operators of two interacting spins. Besides, in order to this system be capable to accomplish a universal operation, it is necessary and sufficient that the evolution of this system be capable to entangle an initial product state [円. Once this entanglement characteristic is present in the evolution of systems described by the Hamiltonian (1.1), the study of the exact solutions of this equation represents an important problem in the analysis of the universal quantum gates and, especially, in the manipulation of quantum dots [1].

In this work, we consider two and three-qubit spin systems in the parallel magnetic fields and present some of their exact solutions. In addition, we discuss an analogue of the Rabi problem for three-spin system.

\subsection{General}

A one-dimensional chain of $n$ interacting spins, immerses in a magnetic field $\mathbf{B}$ and coupled with neighborhoods by a Heisenberg interaction, can be described by the Hamiltonian

$$
\begin{aligned}
& H=\sum_{i=1}^{n} \mathbf{B}_{i} \Sigma^{i}+\frac{1}{2} \sum_{i=1}^{n-1} J_{i(i+1)} \Gamma^{i(i+1)}+\frac{1}{2} J_{1, n} \Gamma^{1 n}, \\
& \Sigma^{k}=I^{\otimes(k-1)} \otimes \sigma \otimes I^{\otimes(n-k)}, \Gamma^{i j}=\Sigma^{i} \cdot \Sigma^{j},
\end{aligned}
$$

where $I$ is the $2 \times 2$ identity, $I^{\otimes n}$ the tensor product of $n$ matrices $I, \sigma=\left(\sigma_{1}, \sigma_{2}, \sigma_{3}\right)$ are the Pauli matrices, $\mathbf{B}_{i}=\mathbf{B}_{i}(t)$ the intensity of the magnetic field in the $i$-th spin, and $J_{i j}$ are interaction functions between the $i$ and $j$ neighborhoods [5]. The case when $J_{1, n} \neq 0$ represents the identification of the extremes (circular chain) and $J_{1, n}=0$ the linear chain. Some important commutation relations are

$$
\left[\Sigma_{i}^{m}, \Sigma_{j}^{n}\right]=2 i \delta_{m n} \varepsilon_{i j k} \Sigma_{k}^{m},\left[\Sigma_{i}^{m}, \Sigma_{j}^{m}\right]_{+}=2 \delta_{i j}
$$


Although the matrices $\Gamma^{m n}$ do not commute with nether $\Sigma^{m}$ and $\Sigma^{n}$, it is possible to see that

$$
\left[\Gamma^{m n}, \Sigma^{m}+\Sigma^{n}\right]=0
$$

As a consequence, any $\Gamma^{j k}$ and, consequently, the interaction terms in the Hamiltonian (1.1), commute with the rotation $\mathscr{R}_{\mathbf{n}}$ in any direction $\hat{\mathbf{n}}$,

$$
\begin{aligned}
& {\left[\Gamma^{j k}, \mathscr{R}_{\mathbf{n}}\right]=0, \hat{\mathbf{n}}^{2}=1,} \\
& \mathscr{R}_{\mathbf{n}}(\theta)=\exp \left(-\frac{i \theta}{2} \hat{\mathbf{n}} \cdot \sum_{i=1}^{n} \Sigma^{i}\right)=\prod_{i=1}^{n} \exp \left(-\frac{i \theta}{2} \hat{\mathbf{n}} \cdot \Sigma^{i}\right) .
\end{aligned}
$$

In the case of parallel fields, with arbitrary time-dependence, the Hamiltonian $H$ preserves, not only the total angular momentum $\mathbf{J}^{2}$, but also the projection of the angular momentum in the direction of the fields. Due to the rotational invariance (1.4), we can apply a rotation and bring $\hat{\mathbf{n}}$ to the $z$ direction. So, without loose of generality, we can choose $\hat{\mathbf{n}}=(0,0,1)$ and $j_{z}$ as a conserved quantity

$$
\left[\mathbf{J}^{2}, H\right]=\left[j_{z}, H\right]=0, \mathbf{J}_{i}=\frac{1}{2} \Sigma^{i}, \mathbf{J}=\sum_{i=1}^{n} \mathbf{J}_{i}, j_{z}=\frac{1}{2} \sum_{i=1}^{n} \Sigma_{3}^{i} .
$$

So, using as a bases eigenvectors of $j_{z}$ (arranged in a descending order of their eigenvalues), the Hamiltonian $H$ takes a block diagonal form, composed by $n+1$ blocks with the $k$-th block describing a system of $(n ! /(n-k+1) !(k-1) !)$ levels, $k=1, \ldots, n+1$. This procedure permits to reduce the problem of $n$ coupled spins (the corresponding system is $2^{n}$-levels) to a set of $n+1$ uncoupled systems where the most complex element is a $n ! /((n / 2) !)^{2}$-level system.

The case with $\mathbf{B}_{i}=0$, all $J_{i j}=J$ and $J_{1, n} \neq 0$ (closed chain) can be exactly solved, and the general case can be treated using approximation or numerical methods by the help of Bethe ansatz [6]. As was already mention, we are going to study exactly solutions for $n=2$ and $n=3$ and parallel time dependent fields.

\subsection{Two coupled spins}

In the case $n=2$ the Hamiltonian (1.1) reads

$$
\begin{aligned}
& H=B_{1} \Sigma_{3}^{1}+B_{2} \Sigma_{3}^{2}+\frac{1}{2} J_{12} \Gamma^{12}, \\
& \Sigma^{1}=\sigma \otimes I, \Sigma^{2}=I \otimes \sigma, \Gamma^{12}=\Sigma^{1} \cdot \Sigma^{2} .
\end{aligned}
$$

The projection of the angular momentum $j_{z}$ can assume the values $1,0,-1$. So the problem can be reduced to a $2 !=2$-level system. It can be viewed explicitly by writing the eigenvectors of $j_{z}$

$$
\begin{aligned}
& \left|j_{z}=1\right\rangle=|00\rangle,\left|j_{z}=-1\right\rangle=|11\rangle \\
& \left|j_{z}=0\right\rangle=\{|10\rangle,|01\rangle\},|l, k\rangle=|l\rangle \otimes|k\rangle \\
& |0\rangle=\left(\begin{array}{l}
1 \\
0
\end{array}\right),|1\rangle=\left(\begin{array}{l}
0 \\
1
\end{array}\right) .
\end{aligned}
$$


From (11.6) we can see that, in this case, we can arrange the $j_{z}$-base in order that it coincide with the canonical base, and, consequently, the Hamiltonian $H$ is already in the block diagonal form

$$
\begin{aligned}
& H=\left(\begin{array}{ccc}
h_{j_{z}=1} & 0 & 0 \\
0 & h_{j_{z}=0} & 0 \\
0 & 0 & h_{j_{z}=-1}
\end{array}\right), h_{j_{z}=0}=\left(\begin{array}{cc}
B_{-}-\frac{J}{2} & J \\
J & -B_{-}-\frac{J}{2}
\end{array}\right), \\
& h_{j_{z}= \pm 1}=\frac{J}{2} \pm B_{+}, B_{ \pm}=B_{1} \pm B_{2} .
\end{aligned}
$$

The special case of parallel fields was treated in Ref. [2], and in case of circular symmetric fields, i.e.,

$$
\mathbf{B}_{1}=\mathbf{B}_{2}=\left(B \cos \omega t, B \sin \omega t, B_{0}\right), B, B_{0}, \omega=\text { const. }
$$

in Ref. [7]. In fact, the latter case represents a generalization of the Rabi problem for two coupled spins. Here there appear two different resonance frequencies. The above results have a special interest in the description of two coupled quantum dots [2], in particular, in the theoretical modeling of universal quantum gates [8, 1], which are fundamental elements in the construction of quantum computers.

\section{Three coupled spins}

Although all the quantum algorithms can be implemented using only systems with one and two spins, the study of three spins system is a fundamental element in the implementation of error correction algorithms [3]. The Hamiltonian for three spins coupled in a triangular configuration and subjected to a magnetic field in the $z$ direction can be written as:

$$
\begin{aligned}
& H=B_{i} \Sigma_{3}^{i}+\frac{1}{2}\left(J_{12} \Gamma^{12}+J_{23} \Gamma^{23}+J_{13} \Gamma^{13}\right), \\
& \Sigma^{1}=\sigma \otimes I \otimes I, \Sigma^{2}=I \otimes \sigma \otimes I, \Sigma^{3}=I \otimes I \otimes \sigma, \Gamma^{i j}=\Sigma^{i} \cdot \Sigma^{j} .
\end{aligned}
$$

We choose the state space for such a system as the direct-product space of the state spaces of individual spins, i.e., we consider the canonical base $\left|\Theta_{i}\right\rangle(i=1,2,3, \ldots, 8)$ composed by the vectors

$$
\begin{aligned}
\left|\Theta_{1}\right\rangle=|000\rangle, \quad\left|\Theta_{2}\right\rangle=|001\rangle, \quad\left|\Theta_{3}\right\rangle=|010\rangle, \quad\left|\Theta_{4}\right\rangle=|011\rangle, \\
\left|\Theta_{5}\right\rangle=|100\rangle, \quad\left|\Theta_{6}\right\rangle=|101\rangle, \quad\left|\Theta_{7}\right\rangle=|110\rangle, \quad\left|\Theta_{8}\right\rangle=|111\rangle,
\end{aligned}
$$

where the above notation indicates $|i j k\rangle=|i\rangle \otimes|j\rangle \otimes|k\rangle, i, j, k=0,1$, and $\{|0\rangle,|1\rangle\}$ are defined in (1.6). More explicitly

$$
\left|\Theta_{1}\right\rangle=\left(\begin{array}{c}
1 \\
0 \\
0 \\
\vdots \\
0
\end{array}\right),\left|\Theta_{2}\right\rangle=\left(\begin{array}{c}
0 \\
1 \\
0 \\
\vdots \\
0
\end{array}\right), \ldots,\left|\Theta_{8}\right\rangle=\left(\begin{array}{c}
0 \\
0 \\
\vdots \\
0 \\
1
\end{array}\right),
$$

i.e., the $n$-th row of the base vector $\left|\Theta_{n}\right\rangle$ is equal to unity and all other entries are zero. 
For this system the total spin operator (1.5) is $\mathbf{J}=\frac{1}{2}\left(\Sigma^{1}+\Sigma^{2}+\Sigma^{3}\right)$ (we set $\hbar=1$ in this study). The $z$ components of the angular momentum operators $\mathbf{J}_{i}=\frac{1}{2} \Sigma^{i}, i=1,2,3$, satisfy eigenvalues equations

$$
\begin{array}{ll}
J_{1 z}\left|\Theta_{1,2,3,4}\right\rangle=\frac{1}{2}\left|\Theta_{1,2,3,4}\right\rangle, & J_{1 z}\left|\Theta_{5,6,7,8}\right\rangle=-\frac{1}{2}\left|\Theta_{5,6,7,8}\right\rangle, \\
J_{2 z}\left|\Theta_{1,2,5,6}\right\rangle=\frac{1}{2}\left|\Theta_{1,2,5,6}\right\rangle, & J_{2 z}\left|\Theta_{3,4,7,8}\right\rangle=-\frac{1}{2}\left|\Theta_{3,4,7,8}\right\rangle, \\
J_{3 z}\left|\Theta_{1,3,5,7}\right\rangle=\frac{1}{2}\left|\Theta_{1,3,5,7}\right\rangle, & J_{3 z}\left|\Theta_{2,4,6,8}\right\rangle=-\frac{1}{2}\left|\Theta_{2,4,6,8}\right\rangle .
\end{array}
$$

The quantum dynamics of this system is governed by the Schrödinger equation $i \partial_{t}|\Psi\rangle=$ $H|\Psi\rangle$, with

$$
|\Psi(t)\rangle=\sum_{i, j, k=\{0,1\}} \vartheta_{i j k}|i j k\rangle=\sum_{\mu=1}^{8} v_{\mu}(t)\left|\Theta_{\mu}\right\rangle=\left(\begin{array}{c}
v_{1}(t) \\
v_{2}(t) \\
\vdots \\
v_{8}(t)
\end{array}\right)
$$

where $i, j, k=0,1$ and the vector $|\Psi(t)\rangle$ has been wrote as a linear combination of the computational basis (2.1.2.2).

The projection of the angular momentum $j_{z}$ can assume the values $3 / 2,1 / 2,-1 / 2,-3 / 2$. So, the problem can be reduced to two (uncoupled) $3 ! / 2 !=3$-level systems. It can be view explicitly by writing the eigenvectors of $\hat{\jmath}_{z}$ as

$$
\begin{aligned}
& \left|j_{z}=3 / 2\right\rangle=|000\rangle,\left|j_{z}=-3 / 2\right\rangle=|111\rangle, \\
& \left|j_{z}=1 / 2\right\rangle=\{|100\rangle,|010\rangle,|001\rangle\}, \\
& \left|j_{z}=-1 / 2\right\rangle=\{|011\rangle,|101\rangle,|110\rangle\} .
\end{aligned}
$$

In this case, the transformation matrix between the $j_{z}$ and canonical base (2.1,2.2) has the form

$$
T=T^{+}=T^{-1}=\left(\begin{array}{ccc}
I_{3 \times 3} & 0 & 0 \\
0 & \sigma_{1} & 0 \\
0 & 0 & I_{3 \times 3}
\end{array}\right)
$$

and the Hamiltonian assumes the form

$$
T H T=\left(\begin{array}{cccc}
h_{3 / 2} & 0 & 0 & 0 \\
0 & h_{1 / 2} & 0 & 0 \\
0 & 0 & h_{-1 / 2} & 0 \\
0 & 0 & 0 & h_{-3 / 2}
\end{array}\right)
$$


where

$$
\begin{aligned}
h_{1 / 2} & =\left(\begin{array}{ccc}
\mathscr{B}_{01}+\frac{1}{2} \mathscr{J}_{11} & J_{23} & J_{13} \\
J_{23} & \mathscr{B}_{10}-\frac{1}{2} \mathscr{J}_{10} & J_{12} \\
J_{13} & J_{12} & -\mathscr{B}_{11}-\frac{1}{2} \mathscr{J}_{01}
\end{array}\right), \\
h_{-1 / 2} & =\left(\begin{array}{ccc}
\mathscr{B}_{11}-\frac{1}{2} \mathscr{J}_{01} & J_{12} & J_{13} \\
J_{12} & -\mathscr{B}_{10}-\frac{1}{2} \mathscr{J}_{10} & J_{23} \\
J_{13} & J_{23} & -\mathscr{B}_{01}+\frac{1}{2} \mathscr{J}_{11}
\end{array}\right), \\
h_{ \pm 3 / 2} & = \pm \mathscr{B}_{00}+\frac{1}{2} \mathscr{J}_{00},
\end{aligned}
$$

and the notation

$$
\begin{aligned}
\mathscr{B}_{m n} & =B_{1}+(-1)^{m} B_{2}+(-1)^{n} B_{3}, \\
\mathscr{J}_{m n} & =J_{12}+(-1)^{m} J_{13}+(-1)^{n} J_{23},
\end{aligned}
$$

are introduced. The exact solution of the above problem can then be obtained by solving the two uncoupled 3-level problems with an effective Hamiltonian given by $h_{ \pm 1 / 2}$. In what follow we study the above system for some special choice of fields and interaction functions.

\subsection{Symmetric case}

In the symmetric case, i.e., the case when $B_{i}(t) \equiv B(t)$ and $J_{n m}(t) \equiv J(t)$, we have

$$
H=2 B j_{z}+\frac{1}{2} J\left(\Gamma^{12}+\Gamma^{23}+\Gamma^{13}\right) .
$$

In this case, as it follows from (2.5), $h_{1 / 2}=h_{-1 / 2}$, so that the problem is reduced to a 3-level system problem.

Due to relation (1.3), the evolution operator $U_{S}$ in the case under consideration can be written as

$$
\begin{aligned}
& U_{S}=R_{3} \exp \left[-i \frac{J_{I}}{2}\left(\Gamma^{12}+\Gamma^{23}+\Gamma^{13}\right)\right], R_{3}=\exp \left(-i B_{I} \sum_{i=1}^{3} \Sigma_{3}^{i}\right), \\
& J_{I}(t)=\int J(t) d t, B_{I}(t)=\int B(t) d t .
\end{aligned}
$$

The first term in $U_{S}$ is just a rotation in the $z$ direction. Using now relation (1.2) and the definition of $\Gamma^{m n}$ it is possible to see that

$$
\left(\Gamma^{12}+\Gamma^{23}+\Gamma^{13}\right)^{2}=9 \mathbf{I},
$$

where $\mathbf{I}$ is the $8 \times 8$ identity matrix. So the evolution operator assumes the form

$$
\begin{aligned}
& U_{S}(t)=\prod_{k=1}^{3}\left[\cos B_{I}(t)-i \Sigma_{3}^{k} \sin B_{I}(t)\right] \\
& \times\left\{\cos \left[\frac{3}{2} J_{I}(t)\right]-\frac{i}{3}\left(\Gamma^{12}+\Gamma^{23}+\Gamma^{13}\right) \sin \left[\frac{3}{2} J_{I}(t)\right]\right\} .
\end{aligned}
$$


For a system of three coupled quantum dots the interaction function can be controlled by external fields [8]. So, once this function is fixed, the above expression can be used to determine an external field which provides any given quantum state. In particular, one can determine the fields by using a maximally entangled pure state (e.g., a GHZ state [9]). The preparation of such kind of states is a fundamental element in the implementation of error correction algorithms for quantum information process.

\subsection{Analogue of the Rabi problem for three-spin system}

We consider the case when three spins are subject to the Rabi field, i.e., a constant magnetic field $B_{0}$ and perpendicular to it a rotating field,

$$
\mathbf{B}=\left(B \cos \omega t, B \sin \omega t, B_{0}\right) .
$$

In this case, the Hamiltonian assumes the form

$$
H=2 \mathbf{B J}+\frac{1}{2} J\left(\Gamma^{12}+\Gamma^{23}+\Gamma^{13}\right) .
$$

Once rotations commute with the interaction terms, we can use a rotating coordinate system that rotates with the field, similar to the usual Rabi problem [10]. So, after the rotation

$$
\mathscr{R}_{z}(\omega t)=\exp \left(-i \omega t j_{z}\right)
$$

the Hamiltonian becomes

$$
\begin{aligned}
H^{\prime} & =\mathscr{R}_{z}^{-1} H \mathscr{R}_{z}-i \mathscr{R}_{z}^{-1} \dot{\mathscr{R}}_{z}=2 \mathbf{B}^{\prime} \mathbf{J}+\frac{1}{2} J\left(\Gamma^{12}+\Gamma^{23}+\Gamma^{13}\right), \\
\mathbf{B}^{\prime} & =\left(B, 0, B_{0}^{\prime}\right), B_{0}^{\prime}=B_{0}-\frac{\omega}{2} .
\end{aligned}
$$

We can now to align the constant field $\mathbf{B}^{\prime}$ with the $z$ direction, applying a second rotation

$$
\mathscr{R}_{y}(\theta)=\exp \left(-\frac{i}{2} \theta \sum_{i=1}^{3} \Sigma_{2}^{i}\right), \tan \theta=\frac{B}{B_{0}^{\prime}} .
$$

In this new frame we have

$$
\begin{aligned}
& H^{\prime \prime}=\mathscr{R}_{y}^{-1} H^{\prime} \mathscr{R}_{y}=2 \mathbf{B}^{\prime \prime} \mathbf{J}+\frac{1}{2} J\left(\Gamma^{12}+\Gamma^{23}+\Gamma^{13}\right), \\
& \mathbf{B}^{\prime \prime}=\left(0,0, B_{0}^{\prime \prime}\right),\left(B_{0}^{\prime \prime}\right)^{2}=\left(B_{0}^{\prime}\right)^{2}+B^{2},
\end{aligned}
$$

and the new Hamiltonian becomes

$$
H^{\prime \prime}=2 B_{0}^{\prime \prime} j_{z}+\frac{1}{2} J\left(\Gamma^{12}+\Gamma^{23}+\Gamma^{13}\right)
$$

The result coincides with the above considered symmetric case. Thus, the evolution operator of the problem under consideration can be written as

$$
\begin{aligned}
& U\left(t, t_{0}\right)=\mathscr{R}_{z}(t) \mathscr{R}_{y}(\theta) U^{\prime \prime}\left(t, t_{0}\right) \underbrace{\mathscr{R}_{y}^{-1}(\theta)}_{=\mathscr{R}_{y}(-\theta)} \underbrace{\mathscr{R}_{z}^{-1}\left(t_{0}\right)}_{=I^{\otimes 3}} \\
& =\mathscr{R}_{z}(t) \mathscr{R}_{y}(\theta) U^{\prime \prime}\left(t, t_{0}\right) \mathscr{R}_{y}(-\theta),
\end{aligned}
$$


with $U^{\prime \prime}$ given by ${ }^{1}$ the equation

$$
\begin{aligned}
& U^{\prime \prime}=\prod_{k=1}^{3}\left(\cos B_{0}^{\prime \prime} t-i \Sigma_{3}^{k} \sin B_{0}^{\prime \prime} t\right) \times \\
& {\left[\cos \left(\frac{3}{2} J_{I}\right)-\frac{i}{3}\left(\Gamma^{12}+\Gamma^{23}+\Gamma^{13}\right) \sin \left(\frac{3}{2} J_{I}\right)\right] .}
\end{aligned}
$$

Of course the conservation of the projection of the angular momentum takes place only in the rotation frame and not in the original one. However, the total angular momentum $\mathbf{J}^{2}$ is an integral of motion in both frames.

The eigenstates of the operator $\mathbf{J}^{2}$, with eigenvalue $j(j+1)$ and $j=1 / 2$ are

$$
\begin{aligned}
& |1\rangle=\frac{1}{\sqrt{2}}[|110\rangle-|011\rangle],|2\rangle=\frac{1}{\sqrt{2}}[|101\rangle-|011\rangle], \\
& |3\rangle=\frac{1}{\sqrt{2}}[|100\rangle-|001\rangle],|4\rangle=\frac{1}{\sqrt{2}}[|010\rangle-|001\rangle],
\end{aligned}
$$

while for $j=3 / 2$ they read

$$
\begin{aligned}
& \left|j_{z}=-3 / 2\right\rangle=|111\rangle,\left|j_{z}=3 / 2\right\rangle=|000\rangle, \\
& \left|j_{z}=-1 / 2\right\rangle=\frac{1}{\sqrt{3}}[|011\rangle+|101\rangle+|110\rangle], \\
& \left|j_{z}=1 / 2\right\rangle=\frac{1}{\sqrt{3}}[|001\rangle+|010\rangle+|100\rangle] .
\end{aligned}
$$

Using the evolution operator, we can, for example, determine the external field that provides the maximum for the transition amplitude between the states $\left|j_{z}=3 / 2\right\rangle$ and $\left|j_{z}=-3 / 2\right\rangle$. Transition amplitude of these states is

$$
\|\langle 111|U| 000\rangle\|^{2}=\frac{1}{16}(\sin \theta)^{6}[\sin (\alpha)-\sin (3 \alpha)]^{2},
$$

with $U$ given by (2.8) and

$$
\tan \theta=\frac{B}{B_{0}-\omega / 2}, \alpha=\sqrt{\left(B_{0}-\omega / 2\right)^{2}+B^{2}}\left(t-t_{0}\right) .
$$

The resonance of this transition occurs in the usual Rabi frequency

$$
\sin \theta=1 \Rightarrow \omega=2 B_{0},
$$

in the instant

$$
t-t_{0}=\frac{\pi}{2 B} \text {. }
$$

Using the procedure developed in Ref. [7], the above results can be used to determine external fields in order to obtain the maximal probability amplitude for some other states. Of course, due to the momentum angular conservation, these amplitudes are non zero only for states with the same $\mathbf{J}^{2}$. In case of quantum dots, these transitions can be used, for example, in the production of multimode field devices, which have applications in quantum optics (see, e.g., [11]).

\footnotetext{
${ }^{1}$ Here we set $t=t-t_{0}$.
} 


\section{Conclusion}

We show how the general problem of $n$ coupled spin, i.e., a $2^{n}$-level system, can be reduced to $n+1$ uncoupled system where the most complex element is, at most, a $n ! /((n / 2) !)^{2}$-level system. After that, we use this procedure to reduce the problem of three coupled spins, an 8-level system, to a problem of a 3-level system in an effective field. We explicitly construct the evolution operator of the system for two special cases: when the fields and interaction are the same in all the spins (the symmetric case), but with a arbitrary time-dependence in the field, and when the spins are subjected to a circular magnetic field (the Rabi problem). In the first case, the results can be used to establish the conditions of the fields that permit obtain a maximum entangled state (e.g., a GHZ state [9]) from an initial product state, what can be used to determine the best filed configuration to implement an error correction algorithm. The second case can be used to explicitly calculate the resonance frequencies of the system.

\section{References}

[1] G. Burkard, D. Loss and D.P. DiVincenzo, Coupled quantum dots as a quantum gate, Phys. Rev. B 59 (1999) 1829

[2] M.C. Baldiotti, D.M. Gitman, Four-level systems and a universal quantum gate, Ann. Phys. (Berlin) 17, No. 7 (2008) 450

[3] M.A. Nielsen, I.L. Chuang, Quantum Computation and Quantum Information, Cambridge University Press, Cambridge 2000

[4] A. Barenco, C.H. Bennett, R. Cleve, D.P. DiVicenzo, N. Margolus, P.W. Shor, T. Sleator, J. Smolin, H. Weinfurter, Elementary gates for quantum computation, Phys. Rev. A 52 (1995) 3457

[5] W. Heisenberg, Mehrkörperproblem und Resonanz in der Quantenmechanik, Z. Phys. 38 (1926) 441

[6] H. Bethe, Zur Theorie der Metalle. Eigenwerte und Eigenfunktionen der linearen Atomkette, Z. Phys. 71 (1931) 205

[7] V.G. Bagrov, M.C. Baldiotti, D.M. Gitman and A.D. Levin. Two interacting spins in external fields. Four-level systems, Ann. Phys. (Berlin) 16, No. 4 (2007) 274

[8] M.C. Baldiotti, V.G. Bagrov, D.M. Gitman, Two interacting spins in external fields and application to quantum computation, Phys. Part. Nucl. Lett. 6 (2009) 559

[9] D. Greenberger, M.A. Horne, A. Zeilinger, Going beyond Bell's Theorem, in "Bell's Theorem, Quantum Theory, and Conceptions of the Universe”, p. 69, M. Kafatos (Ed.), Kluwer, Dordrecht 1989

[10] I.I. Rabi, N.F. Ramsey, J. Schwinger, Use of Rotating Coordinates in Magnetic Resonance Problems, Rev. Mod. Phys. 26 (1945) 167

[11] S. Papademetriou, S. Chakmakjian, and C. R. Stroud, Jr., Optical subharmonic Rabi resonances, J. Opt. Soc. Am. B 9, No. 7 (1992) 1182 